\title{
Robust PID controller design for processes with stochastic parametric uncertainties
}

\author{
Pham Luu Trung Duong, Moonyong Lee* \\ School of Chemical Engineering, Yeungnam University, Gyeongsan, Republic of Korea
}

\section{A R T I C L E I N F O}

\section{Article history:}

Received 13 June 2011

Received in revised form 8 June 2012

Accepted 14 June 2012

Available online 11 August 2012

\section{Keywords:}

Polynomial chaos

Robust controller design

PID controller

Statistical analysis

Stochastic uncertainty

\begin{abstract}
A B S T R A C T
The stability and performance of a system can be inferred from the evolution of statistical characteristics of the system's states. Wiener's polynomial chaos can provide an efficient framework for the statistical analysis of dynamical systems, computationally far superior to Monte Carlo simulations. This work proposes a new method of robust PID controller design based on polynomial chaos for processes with stochastic parametric uncertainties. The proposed method can greatly reduce computation time and can also efficiently handle both nominal and robust performance against stochastic uncertainties by solving a simple optimization problem. Simulation comparison with other methods demonstrated the effectiveness of the proposed design method.
\end{abstract}

(C) 2012 Elsevier Ltd. All rights reserved.

\section{Introduction}

Most engineering applications involve solving physical problems by converting them into deterministic mathematical models with fixed physical parameters. In reality however, these parameters show some randomness, which influences the behavior of the solution. This randomness is not incorporated into deterministic models. Several probabilistic methods have been developed to include this uncertainty in mathematical models.

A representative traditional probabilistic approach for uncertainty quantification is the Monte Carlo (MC) method [1,2]. Its brute-force implementation involves first generating an ensemble of random realizations with each parameter randomly drawn from its uncertainty distribution. Deterministic solvers are then applied to each member to obtain an ensemble of results. The ensemble of results is then post-processed to estimate relevant statistical properties, such as the mean and standard deviation. The estimation of the mean converges with the inverse square root of the number of runs, making MC methods computationally expensive.

Polynomial chaos (PC) is a modern approach to quantifying uncertainty in system models. A PC expansion, originating from Wiener chaos [3], is a spectral representation of a random process by orthonormal polynomials of a random variable. For PC expansions of infinite smooth functions (i.e., analytic, infinitely differentiable), exponential convergence is expected. Ghanem and

\footnotetext{
* Corresponding author. Tel.: +82 53810 2512; fax: +82 538113262 .

E-mail address: mynlee@yu.ac.kr (M. Lee).
}

Spanos [4] showed that PC is an effective computational tool for engineering purposes. Karniadakis and Xiu [5] further generalized PC for use with non-standard distributions. Puvkov et al. [6] proposed that if the Wiener-Askey polynomial chaos expansion is chosen according to the probability distribution of the random input, then it can be used to construct simple algorithms for the statistical analysis of dynamic systems. Several PC expansion-based methods have been proposed including nonintrusive polynomial chaos expansions [7], the stochastic response surface method [8], and the deterministic equivalent modeling method (DEMM) $[6,9,10]$, also known as the probabilistic collocation method (PCM). Controllers for processes with stochastic uncertainties can be designed based on the statistical characteristics of the response. Each distribution has an associated optimal polynomial, which gives optimal convergence. Given that the PCM can compute response statistics effectively, bi-level approaches to controller design under uncertainty have been pursued [6].

This work proposes an efficient method for robust PID controller design that uses the PCM for processes with stochastic uncertainties. The proposed method greatly reduces computational time for controller design and also efficiently handles both nominal and robust performance against uncertainties.

This paper proceeds as follows: Section 2 briefly introduces the basic concepts and theory of the PC method. The PC method is then applied to the statistical analysis of interval systems and robust PID controller design. In Section 3, robust PID controllers are designed for several representative systems with stochastic uncertainties by judging the statistical characteristics of the response. Simulation study compares the proposed PID controller with other methods. 


\section{Statistical analysis using polynomial chaos theory}

\subsection{Generalized polynomial chaos $(g P C)$ theory}

Consider a control system governed by differential algebraic equations [10]:

$\left\{\begin{array}{l}F\left(t, y, y^{\prime}, \ldots, y^{(l)}, \xi\right)=0 \\ g\left(t_{0}, y\left(t_{0}\right), \ldots, y^{(l)}\left(t_{0}\right), \xi\right)=0\end{array}\right.$

where $\xi=\left(\xi_{1}, \xi_{2}, \ldots, \xi_{N}\right)$ is a random vector of mutually independent random components with probability density functions of $\rho_{i}\left(\xi_{i}\right): \Gamma_{i} \rightarrow \mathbb{R}^{+} ; y$ denotes a state variable.

Thus, the joint probability density of the random vector, $\xi$, is $\boldsymbol{\rho}=\prod_{i=1}^{N} \rho_{i}$, and the support of $\xi$ is $\boldsymbol{\Gamma} \equiv \prod_{i=1}^{N} \Gamma_{i} \in \mathbb{R}^{N}$. The set of onedimensional orthonormal polynomials, $\left\{\phi_{i}\left(\xi_{i}\right)_{m=0}^{d_{i}}\right\}$, can be defined in the finite dimension space, $\Gamma_{i}$, with respect to the weight, $\rho_{i}(\xi)$. Based on the one-dimensional set of polynomials, an $\mathbf{N}$-variate orthonormal set can be constructed with $P$ total degrees in the space $\boldsymbol{\Gamma}$ by using the tensor product of the one-dimensional polynomials, the basis function of which satisfies:

$\int_{\Gamma} \Phi_{m}(\xi) \Phi_{n}(\xi) \boldsymbol{\rho}(\xi) d \xi= \begin{cases}1 & m=n \\ 0 & m \neq n\end{cases}$

Considering a response function $f(y(t, \xi)$ ), with statistics (e.g., mean, variance) of interest; the $\mathbf{N}$-variate Pth order approximation of the response function can be constructed as:

$f_{N}^{P}(y(t, \xi))=\sum_{i=1}^{M} \widehat{f}_{i}(t) \Phi_{i}(\xi) ;$
$M+1=\left(\begin{array}{c}N+P \\ N\end{array}\right)=\frac{(N+P) !}{N ! P !}$

where $P$ is the order of polynomial chaos, and $\widehat{f}_{m}$ the coefficient of the gPC expansion that satisfies (2) as:

$\widehat{f}_{i}=\mathbf{E}\left[\Phi_{i} f(y)\right]=\int_{\Gamma} f(y) \Phi_{i}(\xi) \rho(\xi) d \xi$

where $\mathbf{E}[]$ denotes the expectation operator.

\subsection{Probabilistic collocation for statistical analysis of control systems}

In this work, a probabilistic collocation approach [10] is employed for the statistical analysis of control systems to take advantage of its capability to deal with complex response functions effectively. Its algorithm is briefly:

- Choose a collocation set, $\left\{\xi_{i}^{(m)}, w_{i}^{(m)}\right\}_{m=1}^{q_{i}}$, for each random component, $\xi_{i}$, for every direction $i=1, \ldots, N$, and construct a one-dimensional integration rule:

$Q_{q_{i}}^{(i)}[g]=\sum_{j=1}^{q_{i}} g\left(\xi_{i}^{(j)}\right) w_{i}^{(j)}$

where $Q$ [] denotes the quadrature approximation of the univariate integration, $g()$ is a response function of system state, statistics of which are needed to estimate; $w_{i}^{(j)}$ and $\xi_{i}^{(j)}$ are the $j$ th weight and node taken from the collocation set for random component $\xi_{i}$. A Gaussian quadrature (or a Gaussian collocation set) [11] is usually used as the one-dimensional integral rule in classical spectral methods such as the PCM (DEMM).

- Obtain an $\mathbf{N}$-dimensional integration rule (cubature nodes and weights) by the tensorization of the one-dimensional integral rule:

$$
\begin{aligned}
& \ell^{Q}[g]=\left(Q_{q_{1}}^{(1)} \otimes \cdots \otimes Q_{q_{N}}^{(N)}\right)[g] \\
& \quad=\sum_{j_{1}=1}^{q_{1}} \ldots \sum_{j_{N}=1}^{q_{N}} g\left(\xi_{j}^{\left(j_{1}\right)}, \ldots, \xi_{j}^{\left(j_{N}\right)}\right)\left(w_{1}^{\left(j_{1}\right)} \ldots w_{1}^{\left(j_{N}\right)}\right) \simeq \int_{\Gamma} g(\xi) \rho(\xi) d \xi
\end{aligned}
$$

where $\otimes$ denotes the tensor product, and $\ell^{Q}[]$ denotes the multivariate cubature approximation.

- Approximate the gPC coefficients in (4) using the numerical integration rule in (6).

$\widehat{f}_{j}=\ell^{Q}[f(y, \xi) \Phi(\xi) \boldsymbol{\rho}]=\sum_{m=1}^{Q} f\left(\xi^{(m)}\right) \Phi_{j}\left(\xi^{(m)}\right) w^{(m)} \quad$ for $j=1, \ldots, M$

where $\widehat{f}$ represents the numerical approximation of $\widehat{f}$ and $f(\xi) \Phi_{j}(\xi)$ play the role of $g(\xi)$ in Eq. (6).

- Construct an $\mathbf{N}$-variate Pth order gPC approximation of the response function in the form: $\tilde{f}_{N}^{P}=\sum_{j=1}^{M} \widehat{f_{j}} \Phi_{j}(\xi)$.

Once all the gPC coefficients have been evaluated, a postprocessing procedure is then carried out to obtain the statistics of the response function $f(y(t, \xi))$.

The mean value is the first expansion coefficient:

$\mathbf{E}\left[\tilde{f}_{N}^{P}\right]=\mu_{f}=\int_{\Gamma} \tilde{f}_{N}^{P} \rho(\xi) d \xi=\int_{\Gamma}\left[\sum_{j=1}^{M} \widehat{f}_{j}(\xi) \Phi_{j}(\xi)\right] \rho(\xi) d \xi=\widehat{f}_{1}$

The variance of the response function $f(y(t, \xi))$ can be evaluated as:

$$
\begin{aligned}
D_{f}= & \sigma_{f}^{2}=\mathbf{E}\left[\left(f-\mu_{f}\right)^{2}\right]=\int_{\Gamma}\left(\sum_{j=1}^{M} \widehat{f}_{j}(\xi) \Phi_{j}(\xi)-\widehat{f}_{1}\right) \\
& \times\left(\sum_{j=1}^{M} \widehat{f}_{j}(\xi) \Phi_{j}(\xi)-\widehat{f}_{1}\right) \rho(\xi) d \xi=\sum_{j=2}^{M} \widehat{f}_{j}^{2}
\end{aligned}
$$

Eqs. (8) and (9) employ the property that the polynomial set begins with $\Phi_{1}(\xi)=1$. The weight function of the polynomial is the probability density function. If an $f(y)=y$ response function is chosen, the mean and variance of the system's states are approximately given by (8) and (9), respectively. The surrogate gPC series $\tilde{f}_{N}^{P}=\sum_{j=1}^{M} \widehat{f}_{j} \Phi_{j}(\xi)$ can be sampled for obtaining the probability density function for the response function.

The set $\left\{\phi_{i}\right\}_{i=1}^{d_{i}}$ is the orthonormal polynomial of $\xi_{i}$ with weight function $\rho_{i}\left(\xi_{i}\right)$. The weight function is the probability density function of random variable $\xi_{i}$. This links the distribution of random variable $\xi_{i}$ and the type of the orthonormal polynomial in its gPC basis.

\subsection{Polynomial chaos for arbitrary distribution and its associate quadrature}

Let $\rho(\xi)$ be the probability density function of a scalar random variable, $\xi$, which has finite moments of order up to $2 m, m \in \mathbb{N}$. Let $P$ denote the space of a real polynomial, with $P_{m} \subset P$ denoting the space of the polynomial with degree up to $m$. The inner product of two polynomials, $p$ and $q$, relative to $d \lambda=\rho(\xi) d \xi$ is defined by:

$(p, q)_{d \lambda}=\int_{\Gamma} p(\xi) q(\xi) \rho(\xi) d \xi$ 


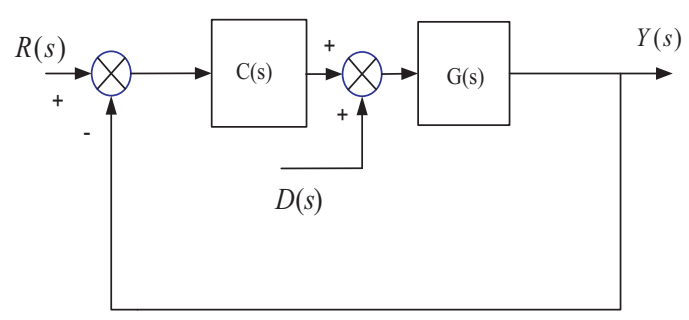

Fig. 1. Closed-loop control system.

where $\Gamma$ is the support domain of the random variable.

Orthonormal polynomials relative to the probability measure, $d \lambda$, can be given by three term recurrence [12]:

$$
\begin{aligned}
& \sqrt{\beta_{k+1}} \phi_{k+1}(\xi)=\left(\xi-\alpha_{k}\right) \phi_{k}(\xi)-\phi_{k-1}(\xi) \sqrt{\beta_{k}}, \quad k=0,1,2 \ldots, \\
& \phi_{-1}(\xi)=0, \quad \phi_{0}(\xi)=\frac{1}{\sqrt{\beta_{0}}}
\end{aligned}
$$

with recurrence coefficients given by:

$$
\begin{aligned}
& \alpha_{k}(d \lambda)=\frac{\left(\xi \phi_{k}, \phi_{k}\right)_{d \lambda}}{\left(\phi_{k}, \phi_{k}\right)_{d \lambda}} \quad k=0,1,2 \ldots, \\
& \beta_{k}(d \lambda)=\frac{\left(\phi_{k}, \phi_{k}\right)_{d \lambda}}{\left(\phi_{k-1}, \phi_{k-1}\right)_{d \lambda}} \quad \beta_{0}=\int_{\Gamma} d \lambda(\xi) \quad k=1,2 \ldots,
\end{aligned}
$$

The index range is infinite $(k \leq \infty)$ or finite $(k \leq m)$ depending on whether the inner product is positive definite on $P$ or $P_{m}$. Hence, the first $m$ recursion coefficient pairs, $\alpha_{k}, \beta_{k}$, are uniquely determined by the first $2 \mathrm{~m}$ moments of $\xi$. Classical methods such as Chebyshev's, for obtaining the recurrence coefficients are based on moments. However, in general obtaining these coefficients from moments becomes severely ill-conditioned and is thus not useful even for well-behaved measures for which there are no classical orthogonal polynomials. Alternatively, approximate methods based on the discretization of an arbitrary measure (e.g. the discretization procedure) can be employed to obtain the recursion coefficients. Discretization is based on approximating the inner product in (10) with a discrete measure:

$(p, q)_{d \lambda}=\int_{\Gamma} p(\xi) q(\xi) \rho(\xi) d \xi=(p, q)_{d \lambda_{S}}=\sum_{\nu=1}^{S} w^{(v)} p\left(\xi^{(v)}\right) q\left(\xi^{(v)}\right)(13)$

The number of discrete $S$ point measures can be increased until the recurrence coefficients are achieved at the desired accuracy. The general-purpose Fejer's quadrature [13] can be used for the discretization in (13). Gautschi [12] showed that the performance of discretization can be increased through multi-component discretization. After constructing an orthonormal set with respect to the arbitrary measure, the collocation point and its corresponding weight in the Gaussian quadrature are given by [11]:

$w^{(i)}=-\frac{a_{n+1}}{a_{n} \phi_{n+1}\left(\xi^{(i)}\right) \phi_{n}^{\prime}\left(\xi^{(i)}\right)} \quad i=1, \ldots, n$

where $a_{n+1}$ is the coefficient of $\xi^{n}$ in $\phi_{n}(\xi)$ and $\xi^{(i)}$ denotes the zeros of $\phi_{n}(\xi)$.

\subsection{Robust PID controller design}

Consider the closed loop control system in Fig. 1, where $C(s)$ is a PID controller:

$C(s)=K_{p}+\frac{K_{i}}{s}+K_{d} s$ and $G(s)$ is a process with stochastic parametric uncertainties:

$G(s)=\frac{b_{m} s^{m}+b_{m-1} s^{m-1}+\cdots+b_{0}}{a_{n} s^{n}+a_{n-1} s^{n-1}+\cdots+a_{0}} e^{-L s}$

where $a_{i}, b_{j}, L$ are random variables with given distributions.

The PID controller's parameters are obtained by optimizing the cost function:

$\min _{K p, K i, K d} J=\min _{K p, K i, K d} \int_{0}^{T}\left|M_{e}(t)\right| d t$

subject to a constraint on the variance of the output:

$\max _{0 \leq t \leq T} D_{y}(t) \leq D_{\max }$

where $M$ and $D$ denote the mean and the variance of the signal, respectively.

Since variance is a measure of the variability of a random process, a large variance of system output implies a large deviation from the nominal response under parametric uncertainties, and thus can lead to either very sluggish or oscillatory responses. The upper bound, $D_{\max }$, of the variance of the system output can be considered the maximum allowable sensitivity of system response to the parametric uncertainties. Hence, adjusting $D_{\max }$ allows tradingoff between the robustness of the response to uncertainty and the response speed. The mean error in (17) can be interpreted as the weighted average error for multi-model systems, where more important models are more heavily weighted (with higher value probability densities).

Note that although the global optimal solution is not guaranteed due to its nonlinear property, the constrained optimization problem by (17) and (18) can be readily solved with conventional non-linear programming methods without significant difficulties in convergence. The resulting PID parameters have the property of a finite variance system output and $M_{y}(t)=1$. From the finite of output variance and mean, the Chebychev inequality [14] becomes $\operatorname{Pr}\left\{\left|y(t)-M_{y}(t)\right| \geq \gamma\right\} \leq\left(D_{y}(t)\right) / \gamma^{2}$, for any $\gamma>0$, which indicates that the probability of the deviation from the mean of the system responses is bounded. Thus, although the proposed approach for optimal PID design does not explicitly specify the stabilizing condition, the constraint on the variance takes into account the requirement for stabilizing the system implicitly in stochastic sense. For example, suppose that $D_{y}(t)=0.01 ; \gamma=1$ at steady state, then $\operatorname{Pr}\{|y(t)-1| \geq 1\} \leq\left(D_{y}(t)\right) / 1=0.01$, which implies the probability of the system response being outside the bound defined by $|y(t)-1|=1$ is just $1 \%$ and thus the response is bounded in stochastic sense. Note that the bound derived by the Chebychev inequality is expected to be somewhat conservative. More accurate bound on the system output can be found by constructing the probability density function (pdf) of the output using either the MC method or the proposed PCM method.

The overall procedure and algorithms for the proposed design method are summarized in Fig. 2.

Note that $D_{\max }$ is selected by trial and error, and the optimization problem may become infeasible depending on the choice of $D_{\max }$. Moreover, it is assumed that the PID parameters are in bounded intervals as in the other existing design methods [14,15] using the scenario approach (Monte Carlo) for stochastic uncertainties. A crude approximation for those bounded intervals can be obtained from the recent stabilization algorithm for arbitrary system [16-18].

\section{Examples}

The performance of the proposed robust PID controller design method was evaluated through case studies. 


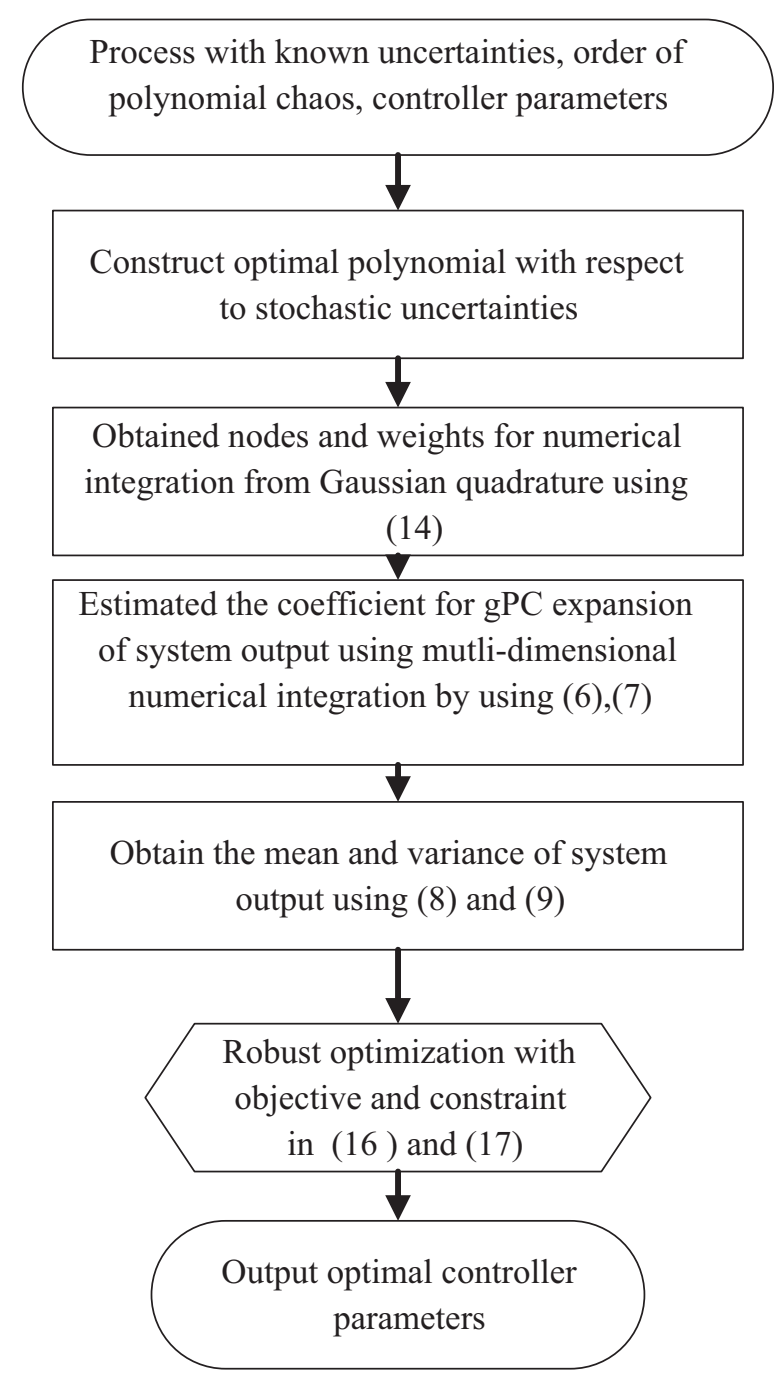

Fig. 2. Flowchart of the robust optimal controller design using polynomial chaos.

\subsection{Case 1}

To demonstrate the proposed method based for statistical analysis, the following first order plus dead-time system of the heated tank in [19] is considered. It has the closed loop configuration outlined in Fig. 1.

$G(s)=\frac{1}{T s+1} e^{-L s}$

where $T, L$ are random variables with triangular distributions $\operatorname{Tr}(0.5,1,1.5)$ and $\operatorname{Tr}(0,0.25,0.5)$, respectively. The triangular distribution is given by the following cumulative distribution function [20]:

$\operatorname{Tr}(a, c, b)= \begin{cases}\frac{(x-a)^{2}}{(b-a)(c-a)} \quad \text { if } \quad a \leq x \leq c \\ 1-\frac{(b-x)^{2}}{(b-a)(b-c)} \quad \text { if } c \leq x \leq b\end{cases}$

with a PI controller $C(s)=2.5+1.67 / s$.

The step input $R(t)=1$ is introduced to the system at $t=0$. The PCM approach is compared with an MC method for predicting output mean and variance for a step reference input (Fig. 3). The two orthonormal polynomial sets with respect to $\operatorname{Tr}(0.5,1,1.5)$ and $\operatorname{Tr}(0,0.25,0.5)$ are obtained using (11) with recurrence coefficients given in Table 1 . The recurrence coefficients for the orthogonal
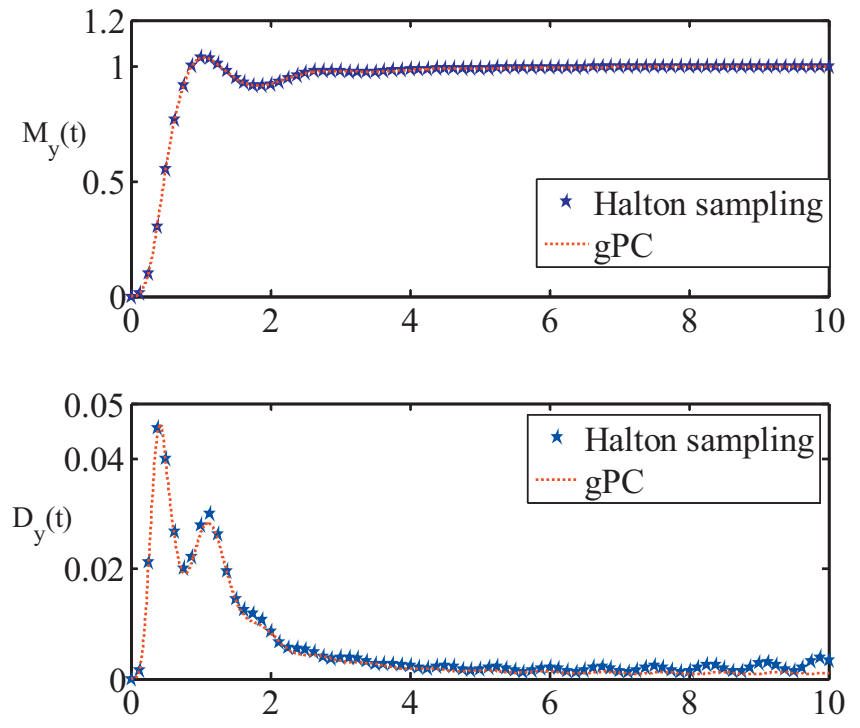

Fig. 3. Statistical characteristics in case 1 .

polynomial sets with respect to these triangular distributions are computed with the $m$ routine $m c d i s . m$ [12], which realizes the algorithm described in (11)-(13) in Section 2. The nodes and weights of cubature are calculated using the DEMM library as in (14) [6]. The coefficients of gPC expansion are estimated from (7) by utilizing the nodes and weights. An analytical representation of the output is then effectively obtained in terms of $\xi=\left(\xi_{1}, \xi_{2}\right)=(T, L)$ as $\tilde{y}_{N}^{P}(t)=\sum_{j=1}^{M} \hat{y}_{j}(t) \Phi_{j}(\xi)$. The probability density function of output can be obtained by a simple sampling of this analytical representation. Note that in the MC method one needs to solve the dynamic equation (1) thousand times at sampling points for obtaining the required probability density, while in the PCM method only at a small number of collocation nodes for obtaining the analytical representation. In the PCM method, the collocation nodes play the same role as the sample points in the MC method. The mean and variance are efficiently estimated by (8) and (9) from the coefficients of the gPC expansion. For a system with a smooth response, exponential convergence is expected for the PCM method [10]. It is found from extensive simulation study that in general only four terms are sufficient in each random variable for presenting the random output. This fast convergence property of the PCM can also be found in $[10,21]$. However, in this study, for guaranteeing the accuracy required for optimization, 15 terms were used for the time delay while only 5 terms for presenting the random time constant, which led to 75 collocation nodes. The probability density functions of output at $0.4 \mathrm{~s}$, where the peak of the variance occurs, obtained by the PCM and MC methods are shown in Fig. 4. The computation times required by both methods to obtain the statistical characteristics and simulation parameters are listed in Table 2 . The proposed method based on the PCM is more efficient than the MC method, while the generated statistical characteristics from both methods

Table 1

Recurrence coefficients for polynomial chaos with respect to $\operatorname{Tr}(0.5,1,1.5)$ and $\operatorname{Tr}(0$ $0.25,0.5)$.

\begin{tabular}{clllll}
\hline$j$ & \multicolumn{2}{l}{$\rho_{T}=\operatorname{Tr}(0.5,1,1.5)$} & & \multicolumn{2}{l}{$\rho_{L}=\operatorname{Tr}(0,0.25,0.5)$} \\
\cline { 2 - 2 } \cline { 5 - 6 } & $\alpha_{i}$ & & & $\alpha_{i}$ & $\beta_{j}$ \\
\hline 0 & 1 & 1 & 0.25 & 1 \\
1 & 1 & 0.0417 & 0.25 & 0.0104 \\
2 & 1 & 0.0583 & 0.25 & 0.0146 \\
3 & 1 & 0.0582 & 0.25 & 0.0145 \\
4 & 1 & 0.0611 & 0.25 & 0.0153 \\
\hline
\end{tabular}




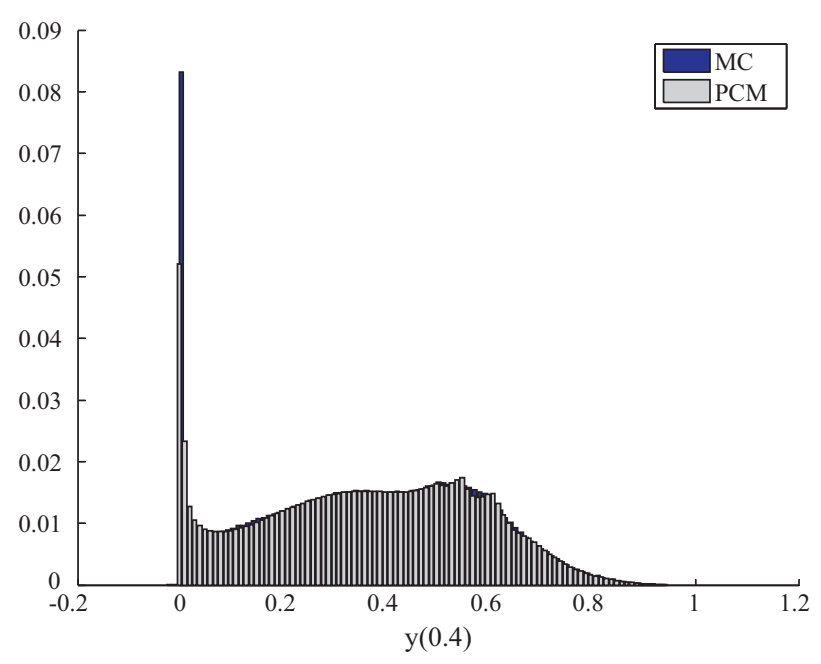

Fig. 4. Probability density function of system output at $0.4 \mathrm{~s}$ for case 1 .

Table 2

Simulation parameters and time profiles for obtaining the statistical characteristic for the PCM and the Monte Carlo (MC) method in case 1.

\begin{tabular}{llll}
\hline $\begin{array}{l}\text { Simulation } \\
\text { parameters }\end{array}$ & PCM & $\begin{array}{l}\text { Computation } \\
\text { time }\end{array}$ & \\
\hline $\begin{array}{l}\text { Quasi Monte Carlo } \\
\text { (Halton Samling) }\end{array}$ & $\begin{array}{l}\text { Quasi Monte Carlo } \\
\text { (Halton Samling) }\end{array}$ & PCM \\
\hline 8100 samples & $\begin{array}{l}75 \text { collocation } \\
\text { points }\end{array}$ & $515.7 \mathrm{~s}$ & $5.9 \mathrm{~s}$ \\
\hline
\end{tabular}

are almost identical. This demonstrates that the PCM gives similar accuracy with far less computational effort than the MC method.

\subsection{Cases 2 and 3}

\subsubsection{Case 2}

The algorithm in Section 2 is applied to the design of robust controllers for the FOPDT system with stochastic uncertainties in the above example.
Optimal PID parameters for robust design are obtained by minimizing the objective function:

$\min _{K p, K i, K d} J=\min _{K p, K i, K d} \int_{0}^{T}\left|M_{e}(t)\right| d t$

subject to:

$\max _{0 \leq t \leq T} D_{Y}(t) \leq 0.02$

The resulting optimal PI controller is:

$C 1(s)=1.445+1.465 / s$

The mean and variance of the system with the optimal controller C1 are shown in Fig. 5.

\subsubsection{Case 3}

The proposed method is also applied to design a controller for a FOPDT system with $T$ and $L$ uniformly distributed: $T \in U[0.5,1.5], L \in U[0,0.5]$ and the same objective and constraint as in case 2 . The result of the optimization problem is $C 2(s)=$ $0.989+1.104 / s+0.007 s$. The mean and variance of the system with optimal C2 and uniform random uncertainties are also shown in Fig. 5. The stochastic uncertainties in cases 2 and 3 have the same support domain, but the variance of the uniform distribution is larger than that of the triangle distribution. As expected, the optimal controller for uniform stochastic uncertainties is more cautious than that for the triangular distribution in case 2. For comparison, a bounded region obtained from 2000 possible responses of the FOPDT system with controller $\mathrm{C} 1$ and each of the two distributions are shown in Fig. 6. Fig. 7 shows a bounded region of output for 2000 possible responses of stochastic FOPDT systems with controller C2. Fig. 6 shows that the optimal controller $\mathrm{C} 1$ designed for the triangular distribution suffers from performance degradation because triangular distributions assume that parameters with values near the peak of the distribution are more heavily weighted in (21). The C2 controller, designed for uniform distributions, can behave well for triangular distributions, as expected. By minimizing the objective function (21), the $\mathrm{C} 1$ controller treats the nominal value as the most probable value, leading to better nominal performance, demonstrated by the mean of the system output in Fig. 5. The $\mathrm{C} 2$ controller tends to treat all the values inside the support domain
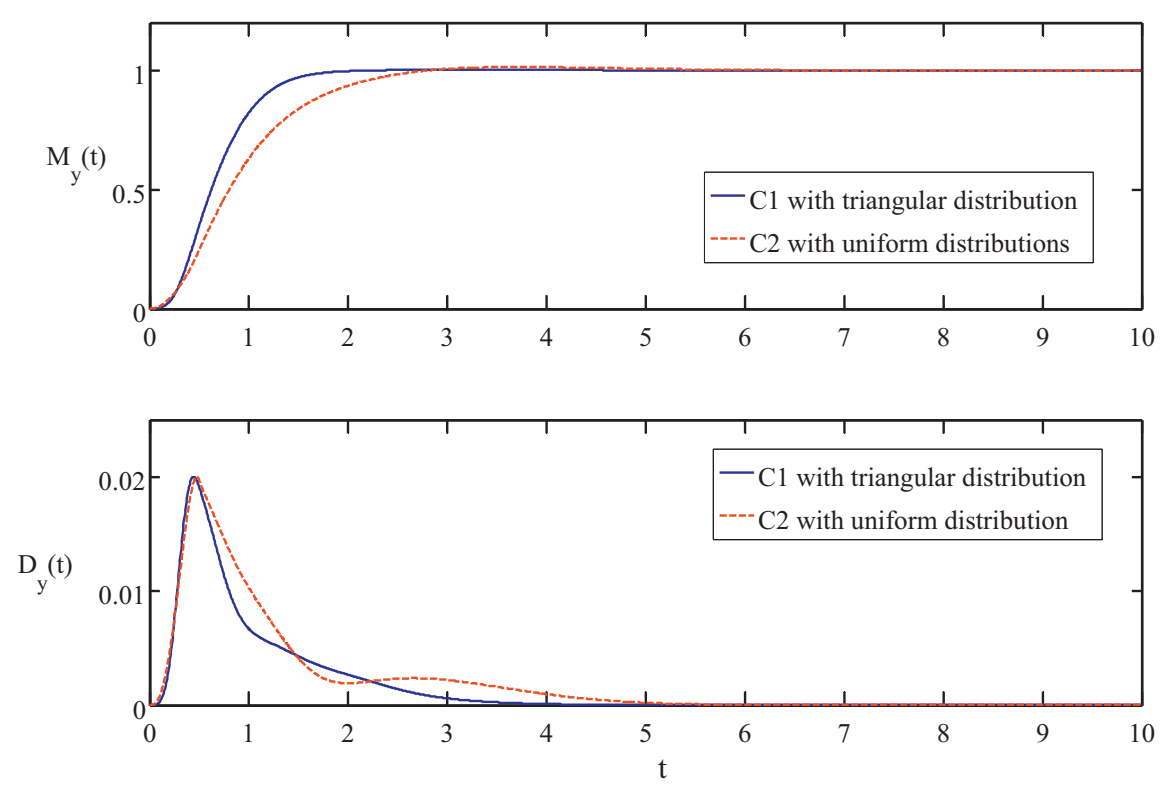

Fig. 5. Mean and variance of system in cases 2 and 3 with their corresponding optimal controllers. 


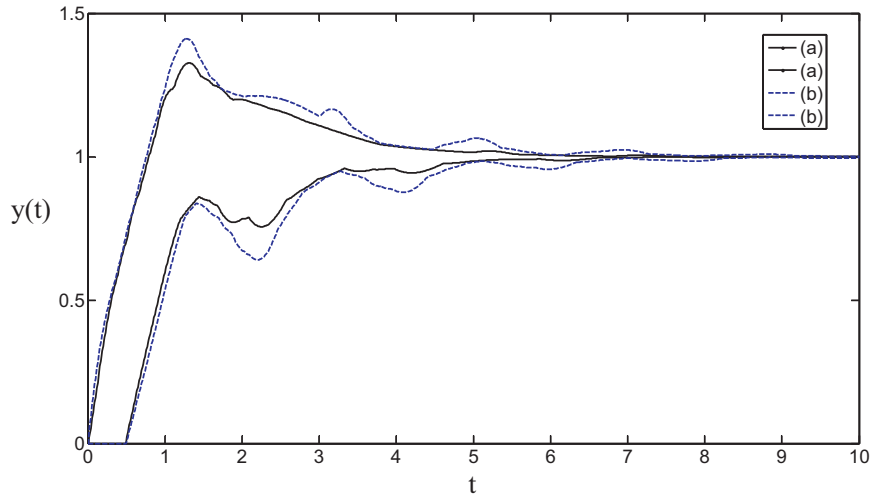

Fig. 6. Bounded region obtained from 2000 possible responses of the uncertain FOPDT systems with the $C 1$ controller. (a) $T$ and $L$ are of triangular distributions; (b) $T$ and $L$ are of uniform distributions.

the same by minimization of the objective function (21), leading to more robust performance.

\subsection{Case 4}

The proposed method is applied to the interval system studied in [22]:

$$
\begin{gathered}
G(s)=\frac{1}{a_{2} s^{2}+a_{1} s+a_{0}} \text { where } 1.8 \leq a_{2} \leq 4.2, \quad 0.8 \leq a_{1} \leq 1.2, \\
1.4 \leq a_{0} \leq 2.6
\end{gathered}
$$

The optimal PID parameters for robust design are obtained by minimizing the objective function:

$\min _{K p, K i, K d} J=\min _{K p, K i, K d} \int_{0}^{T}\left|M_{e}(t)\right| d t$

subject to:

$\max _{0 \leq t \leq T} D_{Y}(t) \leq 0.015$

Fig. 8 compares 2000 possible responses of the uncertain feedback system using a constrained optimal PID controller $\left(K_{p}=\right.$ 54.0; $K_{i}=38.0 ; \quad K_{d}=120.0$ ) obtained by solving (25) and (26) and an unconstrained optimal PID controller $\left(K_{p}=125.6 ; K_{i}=\right.$ 256.3; $K_{d}=383.2$ ) obtained by considering (25) only. The space of stabilization set for PID parameters is unlimited in this example case. To find the optimum parameters of PID control, the search

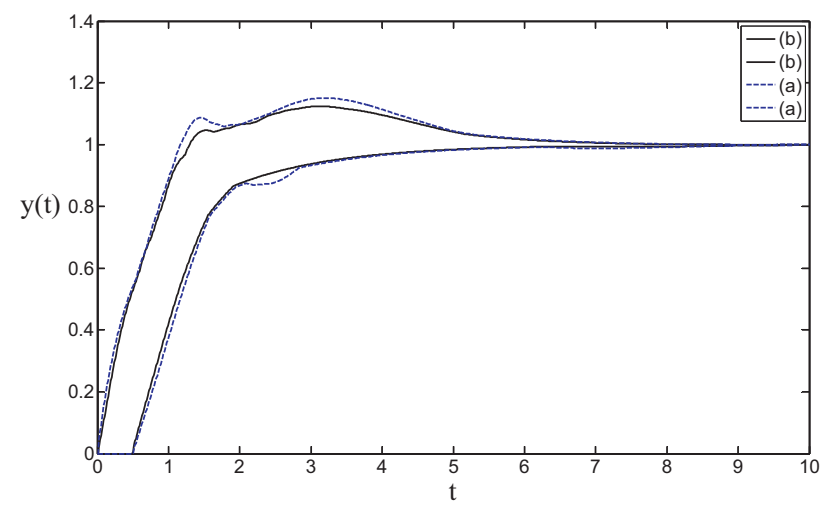

Fig. 7. Bounded region obtained from 2000 possible responses of the uncertain FOPDT system with the C2 controller. (a) $T$ and $L$ are of uniform distributions; (b) $T$ and $L$ are of triangular distributions.
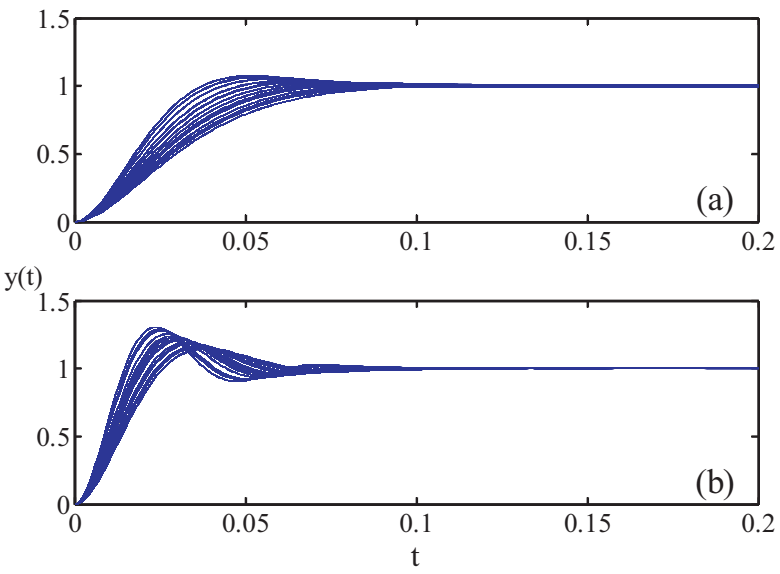

Fig. 8. 2000 possible responses of the uncertain system in case 4 using: (a) The constrained optimal PID controller employing (25) and (26); (b) the unconstrained optimal PID controller employing (25) only.

space was limited to the box $[0,500]^{3}$ for sake of simplicity as in other works based on probabilistic approach [14,15]. The mean and variance of the system outputs in both cases are shown in Fig. 9. More robust and insensitive responses can be obtained from the constrained optimal PID controller by taking output variance into account during controller design; however, this is at the expense of response speed.

The same process model, though with an actuator saturation of $|u(t)| \leq 100$, is considered to evaluate the performance of the proposed PID controller with actuator saturation. For this, a PID controller with a back-calculation structure [23] is employed. The same procedure can be applied for finding the optimal PID parameters and back-calculation coefficient. The resulting PID parameters are $K_{p}=252.3 ; K_{i}=66.1 ; K_{d}=71.8$, with a back-calculation coefficient of $1 / T_{t}=0.3$. Fig. 10 depicts 2000 possible responses from the proposed PID controller with back calculation. The means and variances of system output from these controllers are depicted in Fig. 11, which shows that the constraint on the variance of system output is not activated; hence, the result is equivalent to minimization of the objective function (25) only.
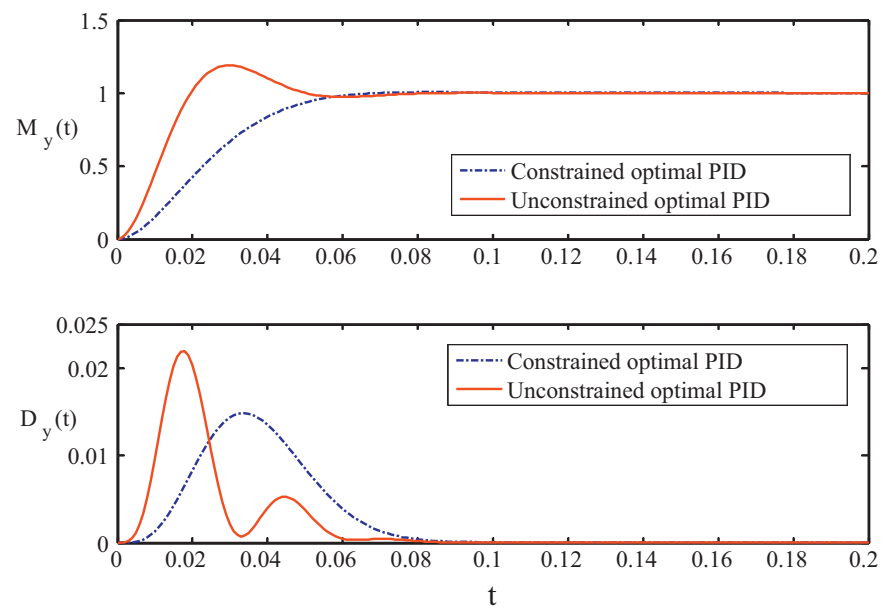

Fig. 9. Mean and variance of output in case 4 . 


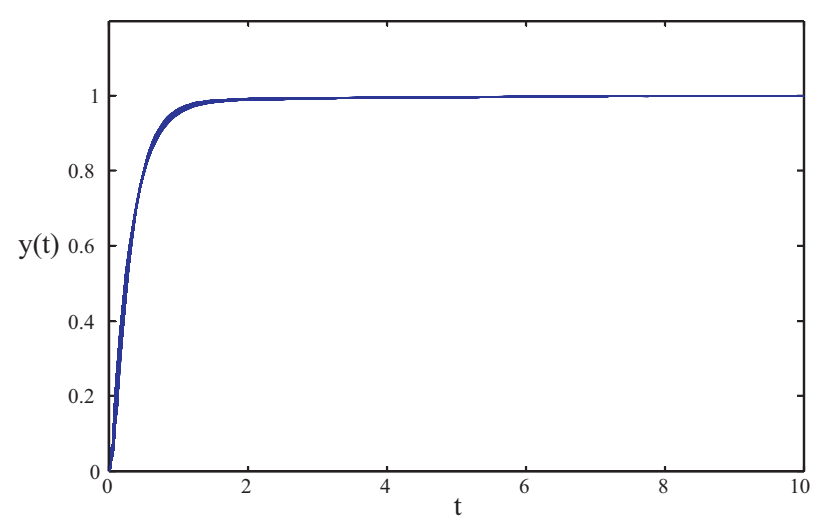

Fig. 10. 2000 possible responses of the uncertain system in case 4 with a controller output saturation of $|u(t)| \leq 100$.

\subsection{Case 5}

The following high order interval system [17] is considered:

$$
\begin{gathered}
G(s)=\frac{5.2(s+2)}{s\left(s^{3}+a_{2} s^{2}+a_{1} s+a_{0}\right)} \quad \text { where } 3.5 \leq a_{2} \leq 4.8, \\
12 \leq a_{1} \leq 15, \quad 9.5 \leq a_{0} \leq 11.5
\end{gathered}
$$

A constrained optimal PID controller can be designed by minimizing the objective function:

$\min _{K p, K i, K d} J=\min _{K p, K i, K d} \int_{0}^{T}\left|M_{e}(t)\right| d t$

subject to

$\max _{0 \leq t \leq T} D_{Y}(t) \leq 0.009$

To evaluate its performance, the proposed optimal PID controller $\left(K_{p}=2.006 ; \quad K_{i}=0.002 ; \quad K_{d}=1.846\right)$ is compared with other existing PID controllers: one $\left(K_{p}=1.72 ; K_{i}=0.05 ; K_{d}=0.01\right)$ designed based on Kharitonov theorem [17] and another $\left(K_{p}=\right.$ $\left.1.6909 ; K_{i}=0.5791 ; K_{d}=0.7827\right)$ from the mixed $H_{\infty} / H_{2}$ method [24].
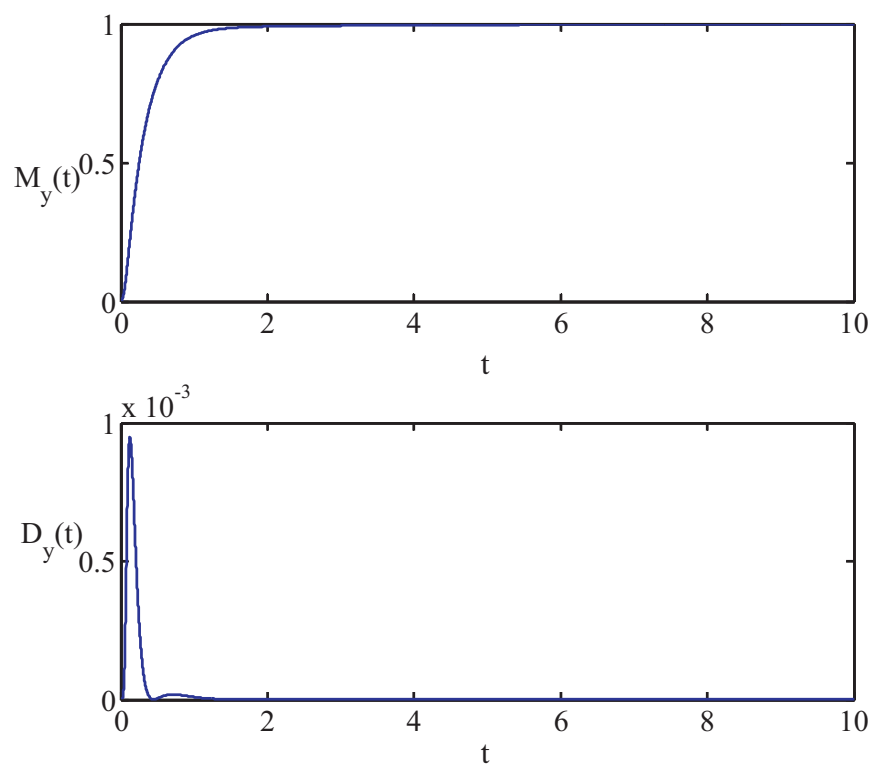

Fig. 11. Mean and variance of output in case 4 with a controller output saturation of $|u(t)| \leq 100$.
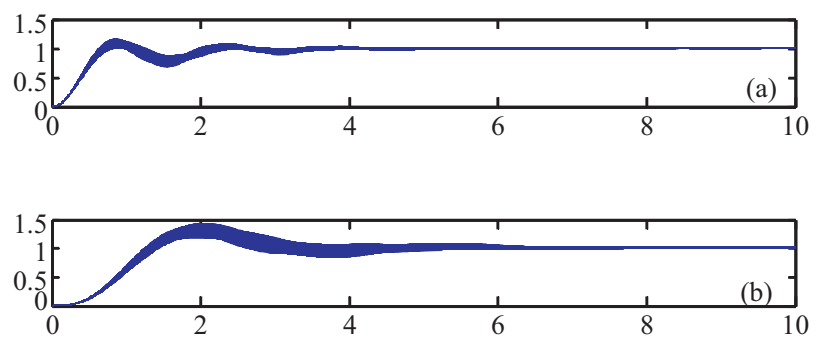

$\mathrm{y}(\mathrm{t})$
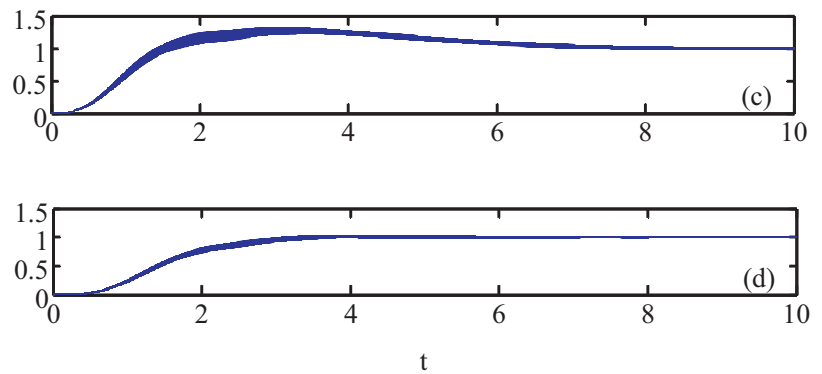

Fig. 12. 1000 possible responses of the uncertain system in case 5 using: the proposed controller (a) without, and (d) with a set-point filter; and the controllers of (b) Huang et al., and (c) Goncalves et al.

Fig. 12 compares 1000 possible responses by each PID controller. The means and the variances of the system output from these methods are shown in Fig. 13. The proposed optimal PID controller gives the most robust and fastest closed-loop responses. A set-point filter can be used if oscillation of the response needs to be mitigated. An optimal PID controller with a second order set-point filter $(\alpha s+1) /\left(\beta_{2} s^{2}+\beta_{1} s+1\right)$ can be designed by the same procedure. The optimal parameters are $\left(\alpha=0.0809 ; \beta_{1}=1.6184 ; \beta_{2}=\right.$ $0.5897 ; K_{p}=3.4520 ; K_{i}=2.1724 ; K_{d}=1.1748$ ). The proposed PID controller with the set-point filter gives smoother responses and is less influenced by parameter variations.

\subsection{Case 6}

In this example, a regulatory case, which is particularly important in process control, is considered. The plant is taken from case 3 (the time delay and time constant are of uniform distributions) for a robust performance design as discussed in case 3 . The system
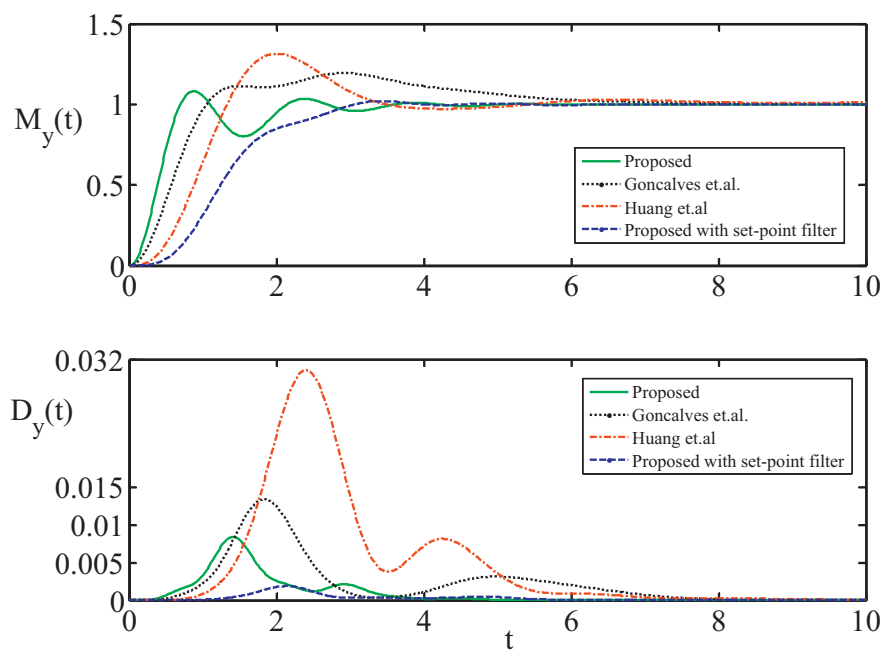

Fig. 13. Mean and variances of output in case 5 . 

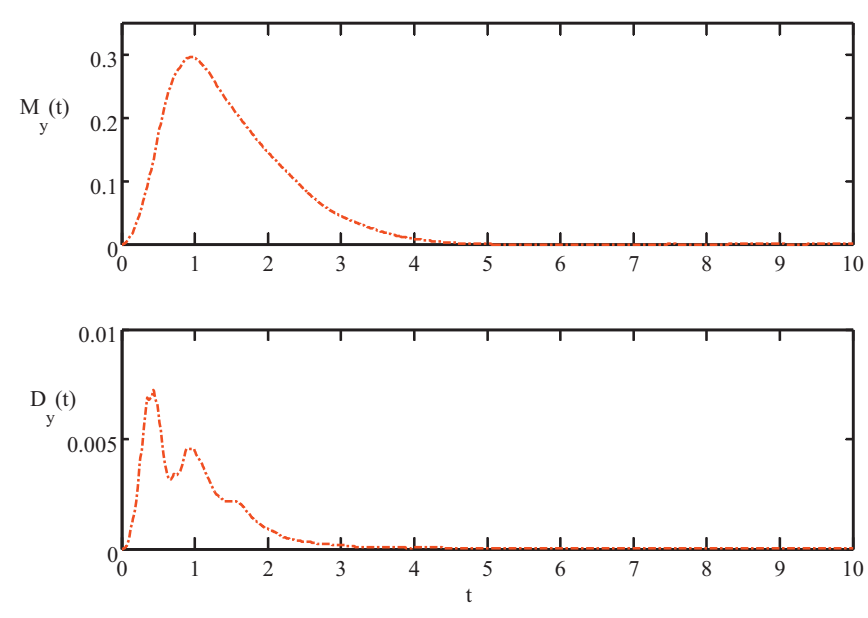

Fig. 14. Mean and variance of output in case 6.

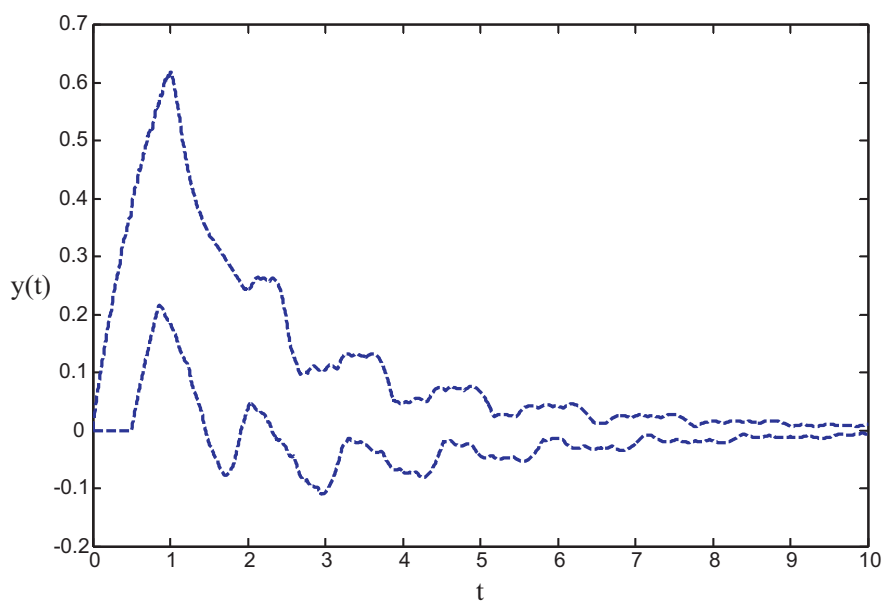

Fig. 15. Bounded region obtained from 2000 possible responses of the uncertain FOPDT in case 6.

is now subjected to input disturbance $D(t)=1$ at $t=0$, while $R(t)=0$. The optimization problem is:

$\min _{K p, K i, K d} J=\min _{K p, K i, K d} \int_{0}^{T}\left|M_{e}(t)\right| d t$

subject to:

$\max _{0 \leq t \leq T} D_{Y}(t) \leq 0.01$

The resulting optimal parameters are: $K_{p}=1.957 ; K_{i}=$ $2.012 ; K_{d}=0.32$. The mean and variance with this controller is shown in Fig. 14. Fig. 15 shows a bounded region for 2000 possible responses of uncertain system with the proposed controller. It can be seen from the response of the uncertain systems that the proposed control still can achieve a satisfactory response for the regulatory case.

\section{Conclusions}

A robust PID controller was designed and its statistical analysis was studied for processes with stochastic parametric uncertainties. The use of the polynomial chaos method greatly reduced computation time over the traditional Quasi Monte Carlo method, without a loss of accuracy. The stability of uncertain systems can be inferred from the statistical evolution of the system output, and hence robust PID controllers can be efficiently designed with respect to stochastic uncertainties. Depending on whether robustness or nominal performance is of primary concern, different distribution can be assumed for the stochastic uncertainties. With different assumptions of the distribution of uncertainties, optimal PID parameters were computed using a simple nonlinear optimization. Several simulation examples showed that the proposed design method gives robust closed-loop responses for various stochastic systems.

\section{Acknowledgment}

This research was supported by the Human Resource Development Program of Korea Institute of Energy Technology Evaluation and Planning (KETEP) grant (No. 20104010100580) funded by the Korean Ministry of Knowledge Economy.

\section{References}

[1] K.A. Puvkov, N.D. Egupov, Classical and Modern Theory of Control System, vol 2, BMGTU, Moscow, 2003 (in Russian).

[2] J.S. Liu, Monte Carlo Strategies in Scientific Computing, Springer-Verlag, New York, 2001.

[3] N. Wiener, The homogeneous chaos, American Journal of Mathematics 60 (1938) 897-936.

[4] R.G. Ghanem, P.D. Spanos, Stochastic Finite Elements: A Spectral Approach, Dover Publications, New York, 1991.

[5] D. Xiu, G.R. Karniadakis, The Wiener-Askey polynomial chaos for stochastic differential equations, SIAM Journal on Scientific Computing 24 (2002) 619-644.

[6] K.A. Puvkov, N.D. Egupov, A.M. Makarenkov, A.I. Trofimov, Theory and Numerical Methods for Studying Stochastic Systems, Fizmatlits, Moscow, 2003 (in Russian).

[7] S. Hosder, R.W. Walters, R.A. Perez, A non-intrusive polynomial chaos method for uncertainty propagation in CFD simulation, in: Proceeding of 44th AIAA Aerospace Sciences Meeting and Exhibit, 2006.

[8] S.S. Isukapalli SS, A. Roy, P.G. Georgopoulos, Efficient sensitivity/uncertainty analysis using the combined stochastic response surface method, and automated differentiation: application to environmental and biological systems, Risk Analysis 20 (2000) 591-602.

[9] M. Tatang, Direct incorporation of uncertainty in chemical and environmental engineering systems, Ph.D. Thesis, Massachusetts Institute of Technology 1995.

[10] D. Xiu, Numerical Method for Stochastic Computations: A Spectral Method Approach, Princeton University Press, 2010.

[11] P.K. Kythe, M.R. Schaferkotter, Handbook of Computational Method for Integration, Chapman \&Hall/CRC Press, Boca Raton, 2005.

[12] W. Gautschi, Orthogonal Polynomials: Computation and Approximation, Oxford University Press, 2004.

[13] L. Fejér, Mechanische Quadraturen mit positiven Cotesschen Zahlen, Mathematische Zeitschrift 37 (1933) 287-309.

[14] R. Tempo, G. Caliafore, F. Dabbene, Randomized Algorithm for Analysis and Control of Uncertain Systems, Springer-Verlag, London, 2005.

[15] T. Alamo, R. Tempo, E.F. Camacho, Randomize algorithm for probabilistic solution of uncertain feasibility and optimization problem, IEEE Transactions on Automatic Control 54 (11) (2009) 2545-2559.

[16] T. Liang, J. Chen, C. Lei, Algorithm of robust stability region for interval plant with time delay using fractional order $\mathrm{PI}^{\lambda} \mathrm{D}^{\lambda}$ controller, Communications in Nonlinear Science and Numerical Simulation 17 (February (2)) (2012) 979-991.

[17] Y.J. Huang, Y.J. Wang, Robust PID tuning strategy for uncertain plants based on the Kharitonov theorem, ISA Transactions 39 (2000) 419-431.

[18] T. Emami, J.M. Watkin, Weighted sensitivity design of PID controller for an arbitrary order transfer function with time delay, in: Proceeding of the 4th Annual GRASP Symposium, Wichita State University, 2008.

[19] J.E. Normey Rico, E.F. Camacho, Control of Dead-time Process, Springer-Verlag, London, 2007.

[20] C. Forbes, M. Evans, N. Hasting, B. Peacok, Statistical Distribution, John Wiley \& Sons, Hoboken, 2011.

[21] Z.K. Nagy, R.D. Braatz, Distributional uncertainty analysis using power series and polynomial chaos expansions, Journal of Process Control 17 (2007) 229-240 (Special Issue on Advanced Control of Chemical Processes).

[22] D.W. Gu, P.H. Petkov, M.M. Konstantinov, Robust Control Design with MATLAB, Springer-Verlag, London, 2005.

[23] K.J. Astrom, T. Hagglund, Advanced PID Control, ISA - Instrumentation, System, and Automation Society, 2006.

[24] E.N. Goncalves, R.M. Palhares, R.H. Takashi, A novel approach for $\mathrm{H}^{2} / \mathrm{H}_{\infty}$ robust PID synthesis, Journal of Process Control 18 (2008) 19-26. 